$10 \mid 2015$

Inv(i)erno 2015

\title{
Los problemas del concepto de "idealismo trascendental" en el Sistema del idealismo trascendental de F.W.J. Schelling
}

Fernando Wirtz

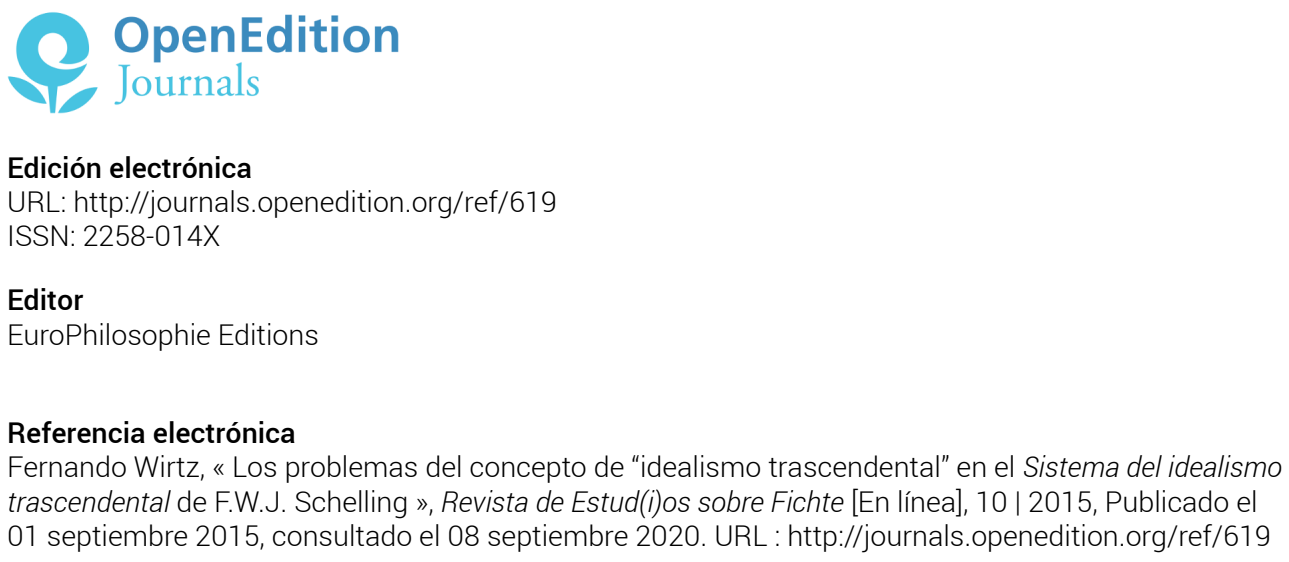

Este documento fue generado automáticamente el 8 septiembre 2020.

(c) EuroPhilosophie 


\section{Los problemas del concepto de "idealismo trascendental" en el Sistema del idealismo trascendental de F.W.J. Schelling}

Fernando Wirtz

"Schelling posee simplemente un concepto de idealismo trascendental distinto que el mío."

Fichte, Manuscrito "Bei der Lectüre von Schellings tr. Idealismus", GA II/5, 414

\section{Introducción}

1 El término "idealismo", que aparece en el título y en el texto de una de las obras consideradas usualmente más importantes del corpus schellingueano, el Sistema del idealismo trascendental (de ahora en adelante, Sistema) de 1800, no resulta tan unívoco como podría parecer. Evidencia de esto no es únicamente la bibliografía secundaria contemporánea que lidia con el sentido de este término, sino también los malos entendidos que se suscitaron entre los coetáneos del filósofo. En sus obras posteriores de 1801, así como en su correspondencia con Fichte, Schelling debe, obligado por la confusión que genera el término, realizar aclaraciones al respecto. ${ }^{1} \mathrm{El}$ presente trabajo se propone formular nuevamente aquella misma pregunta, es decir, ¿qué significa la palabra "idealismo" en el Sistema del idealismo trascendental? La respuesta a este enigma no es clara. No lo fue en la época de Schelling ni lo fue entre sus posteriores comentadores.

2 En los últimos años se ha señalado más de una vez la fricción resultante de la exposición del idealismo trascendental y la filosofía de la naturaleza dentro del Sistema. ${ }^{2}$ En esta tensión juega un papel preponderante la ausencia de una definición unívoca por parte de Schelling del concepto que da el título a su obra. Con el fin de echar luz 
sobre el sentido del término "idealismo", intentaremos colocar el Sistema en un marco textual más amplio, comparándolo con las reflexiones que se encuentran en la correspondencia con Fichte y con los pronunciamientos en dos de sus textos de 1801, Sobre el verdadero concepto de la filosofía de la naturaleza y Presentación de mi sistema de la filosofía.

3 En el presente trabajo se distingirá primero entre dos sentidos diferentes de "idealismo trascendental" que predominan en el texto schellinguiano, uno amplio y uno estrecho (1). Estos dos sentidos, lejos de convivir armónicamente entre sí, parecen engendrar tensiones que tanto Fichte (en sus cartas) como el mismo Schelling (en sus textos de 1801) parecen reconocer. Es por ello que en el segundo apartado (2) se intentará ver de qué forma Schelling articula estos dos sentidos, en base a las dos obras de 1801 ya citadas. La hipótesis de este trabajo es que Schelling, al establecer un concepto estrecho de idealismo trascendental como opuesto a la filosofía de la naturaleza relativiza el alcance y el rol del idealismo dentro de su sistema de la filosofía en general.

\section{Los diferentes sentidos de "idealismo trascendental"}

El primer objetivo de este trabajo es distinguir los sentidos que la palabra "idealismo" presenta en el Sistema del idealismo trascendental. Schelling parece utilizar la expresión "idealismo trascendental" en dos sentidos predominantes, un sentido amplio y un sentido estrecho. Estos dos sentidos engendran una dificultad terminológico-semántica. Para apoyar esta idea el texto se referirá a la correspondencia entre Schelling y Fichte, donde este último da cuenta por sus comentarios de que su joven colega no es del todo unívoco en su enunciación.

Uno de los problemas principales que se plantea el Sistema es cómo conciliar el conflicto aparentemente insalvable entre idealismo y realismo. Este conflicto fue, si no inaugurado, al menos reavivado por Kant. ¿Cómo sostener la centralidad de la subjetividad como condición de posibilidad de la percepción sin reducir lo "externo" a una mera ilusión? En ese sentido, idealismo y realismo son dos posturas filosóficas. A primera vista, Schelling parece colocar estas dos posturas como enfrentadas $y$, de este modo, simétricas. Idealismo y realismo, actitudes contrarias entre sí, se presuponen y complementan mutuamente.

6 Para el autor del Sistema, esta oposición absoluta en el ámbito teórico, se torna relativa en cuanto a su telos común. En las Cartas filosóficas sobre dogmatismo y criticismo (Philosophische Briefe über Dogmatismus und Kriticismus) (1795), Schelling ya afirmaba que ambas posturas filosóficas, una vez que alcanzan su objetivo último, es decir, el conocimiento de lo absoluto, tenderían inevitablemente a identificarse: "ambos [idealismo y realismo] se unifican en lo absoluto, deben pues cesar en su contraposición". ${ }^{3}$ Si en esa obra las dos posturas filosóficas principales en discusión eran el dogmatismo y el criticismo, en el Sistema de 1800 la disyuntiva se complejiza con la introducción de una tercera postura filosófica: la perspectiva "ideal-realista". Esta idea, que en obras anteriores aparecía sugerida pero no tematizada, es utilizada por Schelling, según se verá más adelante, como sinónimo de "idealismo trascendental". El razonamiento de Schelling considera que siendo el absoluto uno e indivisible no pueden existir dos ciencias o posturas filosóficas opuestas de forma inconmensurable. En todo 
caso, estas dos posiciones deben coincidir en algún lugar. Todo el saber requiere una forma sistemática, debe haber una conformidad entre sus partes. Este es un precepto metodológico central para Schelling. Si esto es así, es posible que estas dos posturas filosóficas no deban ser, de forma necesaria, mutuamente excluyentes, al contrario de lo que, a ojos de Schelling, había afirmado Fichte en la Primera introducción a la doctrina de la ciencia ${ }^{4}$. Antes de profundizar en la expresión "idealismo trascendental" es pertinente determinar qué entiende Schelling por "idealismo" a secas en el Sistema del idealismo trascendental.

7 Un primer sentido del término puede caracterizarse como "ingenuo". El idealismo ingenuo es un modo de ver el mundo que postula que la realidad es una representación del yo individual, que todo es apariencia (Schelling lo llama en el Sistema, "idealismo impropio", "uneigentliche Idealismus")..$^{5}$ De este mismo modo es posible hablar de un "realismo ingenuo" que rezaría simplemente: "hay cosas fuera de nosotros". ${ }^{6}$ Para esta perspectiva, el yo es una cosa entre las cosas y, por lo tanto, recibe todas las determinaciones que pueden afectar a los objetos sensibles.

8 Junto al idealismo y al realismo ingenuos podemos distinguir entre un "idealismo filosófico" y un "realismo filosófico", inspirados en sus correlatos intuitivos. A diferencia de sus semejantes ingenuos, el idealismo y realismo filosóficos se preguntan por el fundamento de totalidad. Mientras el realismo filosófico podría definirse como la posición teórica para la cual el ser es lo originario, ${ }^{7}$ para el idealismo filosófico el pensar es lo originario y primero.

9 Estas dos posturas filosóficas funcionan como el punto de partida intuitivo de dos ciencias o sistemas filosóficos complementarios, la filosofía de la naturaleza (realismo) y la filosofía trascendental (idealismo). Para la ciencia lo que importa es establecer como cierto un saber. Este saber sólo es posible allí donde se da la identidad entre lo subjetivo y lo objetivo. Así, Schelling reduce la oposición idealismo-realismo a una cuestión de método. Si se establece lo subjetivo como principio de la filosofía, se estará en el terreno de la filosofía del espíritu o filosofía trascendental. Si se establece lo objetivo como lo prioritario y primero, se estará en el ámbito de la filosofía de la naturaleza. Para demostrar la verdad de estas ciencias debe hacerse coincidir lo subjetivo y lo objetivo, ese es su precepto metodológico. En otras palabras, la filosofía trascendental debe demostrar la objetividad de las representaciones subjetivas, demostrar la identidad entre las representaciones y las cosas. De esta forma, parecería que para Schelling, aquella máxima fichteana por la cual "qué clase de filosofía se elige, depende, según esto, de qué clase de hombre se es" deja de poseer validez porque ambas ciencias coinciden al final de su despliegue. ${ }^{8}$ Ambas ciencias son igual de válidas y complementarias de manera que hasta el realismo spinozista "puede continuar existiendo en cuanto sistema real", aunque sea "sólo como ciencia de la naturaleza". ${ }^{9}$

Hasta aquí pueden distinguirse, por lo tanto, entre tres pares de conceptos que se diferencian cualitativamente entre sí: idealismo ingenuo y realismo ingenuo, idealismo filosófico y realismo filosófico, filosofía trascendental y filosofía de la naturaleza.

11 No obstante, esta simetría relativa entre la filosofía trascendental y la filosofía de la naturaleza parece quebrarse cuando Schelling introduce cierta ambigüedad acerca del término "idealismo trascendental". El problema es el siguiente: mientras que en algunos pasajes Schelling se refiere al "idealismo trascendental" como la ciencia del espíritu o subjetiva (en contraposición a la filosofía de la naturaleza), en otros fragmentos parece referirse a una tercera ciencia. Hay dos sentidos diferentes para la 
expresión "idealismo trascendental": 1) en un sentido amplio esta locución hace referencia al "ideal-realismo"; 2) en un sentido estrecho hace referencia a la ciencia fundamental de la conciencia opuesta como disciplina a la filosofía de la naturaleza. La siguiente cita parece referir al primer sentido (1):

"Si reflexiono meramente sobre la actividad ideal, me surge el idealismo o la afirmación de que el límite es puesto sólo por el Yo. Si reflexiono meramente sobre la actividad real, se me origina el realismo o la afirmación de que el límite es independiente del Yo. Si reflexiono sobre las dos a la vez, entonces me surge un tercero a partir de ambos, lo que se puede llamar ideal-realismo o lo que hasta ahora hemos designado con el nombre de idealismo trascendental." ${ }^{10}$

Un poco antes Schelling había afirmado: "El fin de la presente obra [del Sistema del idealismo trascendental] es precisamente este: ampliar el idealismo trascendental a lo que debe ser en realidad, o sea, un sistema de todo el saber" ${ }^{11}$ Pero, ¿es que acaso el idealismo trascendental es la disciplina más alta de la ciencia o es la ciencia misma como totalidad en su máxima expresión? Por otra parte, ¿no debería ser también el fin de la filosofía de la naturaleza alcanzar la totalidad del saber? Después de todo, ambas ciencias coinciden en su telos. Más adelante escribe el autor:

"Si todo saber se basa en la coincidencia de ambos [de lo subjetivo y lo objetivo], entonces la tarea de explicar esta coincidencia es sin duda la más elevada con respecto a todo saber y si, como se concede generalmente, la filosofía es la más alta y encumbrada de todas las ciencias, ésta es sin duda la tarea principal de la filosofía." ${ }^{12}$

13 A partir de estas citas sería lógico pensar que el idealismo trascendental es el "sistema de todo el saber", esto es, la actividad que reflexiona al mismo tiempo sobre lo subjetivo y lo objetivo, lo ideal y lo real. Hasta aquí, "idealismo trascendental" pareciera ser sinónimo de "ideal-realismo" (lo que llamamos sentido amplio (1)). La confusión surge cuando se contrasta con pasajes como este:

"Con lo dicho hasta aquí no sólo hemos deducido el concepto de filosofía trascendental sino que, al mismo tiempo, hemos proporcionado al lector una visión de todo el sistema de la filosofía, que, como se ve, es realizado por dos ciencias fundamentales, opuestas entre sí en el principio y la dirección, que se buscan y se complementan recíprocamente. Aquí no se ha de exponer todo el sistema de la filosofía, sino sólo una de sus ciencias fundamentales y, en primer lugar, se la ha de caracterizar con mayor precisión conforme al concepto deducido." ${ }^{13}$

14 Esto sugiere que el texto del Sistema del idealismo trascendental expone una de las dos ciencias fundamentales que componen el "sistema de todo el saber", es decir, que el idealismo trascendental no es la totalidad del sistema de todo el saber. Esto estaría de acuerdo con lo que caracterizamos como sentido estrecho (2).

Esta confusión se refuerza si se le presta atención a la correspondencia mantenida entre Schelling y Fichte. En su carta del 15 de noviembre de 1800, Fichte comunica a su colega en Jena su desacuerdo con respecto a la "oposición [Gegensatz] entre Filosofía Trascendental y Filosofía de la Naturaleza"..$^{14}$ Para Fichte, la acción trascendental del Yo absoluto es el fundamento ontológico de la filosofía trascendental y de la filosofía de la naturaleza. Si bien Schelling podría estar en principio de acuerdo con esta proposición, Fichte entiende que su interlocutor piensa la génesis de la naturaleza (de lo objetivo) como algo que se añade (hinzukommen) a lo puramente subjetivo. La naturaleza aparece presentada con cierta autonomía con respecto a la conciencia. Como señala Serrano Marín:

“[...] mientras que para Fichte el Yo es el único incondicionado a partir del cual explicar naturaleza y conciencia, para Schelling tanto filosofía de la naturaleza 
como filosofía trascendental poseen su propio incondicionado, que no es, sin embargo, el incondicionado o el Absoluto mismo, sino éste presentado desde el sujeto o desde el objeto." ${ }^{15}$ hubiera comprendido que se trataba de dos ciencias complementarias que constituían un solo sistema fundamental, ¿no habría formulado su crítica de otro modo? Tal como escribe Fichte, parece evidente que comprendió que se trata únicamente de dos ciencias cualitativamente opuestas y con cierto grado de autonomía ontológica, es decir, lo interpretó en un sentido estrecho (2). De esta forma parece entenderlo también Reinhold en el segundo tomo de sus Contribuciones para un panorama más sencillo del estado de la filosofía (Beyträge zur leichtern Übersicht des Zustandes der Philosophie) (1801). Para él, Schelling liberó a la filosofía de la naturaleza y la elevó al rango de ciencia fundamental, colocándola no por sobre sino junto a la filosofía trascendental. ${ }^{16}$ Eschenmayer también manifiesta su confusión. ${ }^{17}$ Tanto Fichte como Reinhold, pues, entienden a la filosofía de la naturaleza y a la filosofía trascendental como ciencias simétricas $\mathrm{y}$, en ese sentido, enfrentadas. Ciertamente el autor del Sistema no termina de explicitar detalladamente el grado de autonomía de cada una de las ciencias. Si bien resulta lógico que el Sistema del idealismo trascendental en su título haga referencia en su título a la filosofía trascendental en sí misma (y no al ideal-realismo), es importante remarcar que la confusión se basa en la ambigüedad del término y en que Schelling no explica cómo se articula el sentido estrecho con el sentido amplio.

De todas formas, Schelling responde. En la carta del 19 de noviembre de 1800, distingue entre la Wissenschaftslehre de Fichte, la filosofía trascendental y la filosofía de la naturaleza. Para Schelling, la Wissenschaftslehre constituye "la prueba formal del idealismo" porque no llega a suprimir la identidad entre lo subjetivo y lo objetivo. ${ }^{18}$ Esta supresión se evidencia en las dos ciencias del sistema schellinguiano: la filosofía trascendental comienza con lo subjetivo escindido, la filosofía de la naturaleza comienza con lo objetivo escindido. Así, se define a la Wissenschaftslehre como "idealrealista". ${ }^{19}$

Sandkaulen, en su artículo ¿Qué significa idealismo? (Was heißt Idealismus?) plantea el problema de los múltiples sentidos del término en lo que llama el "esbozo de sistema" o "Systemskizze". ${ }^{20}$ Sandkaulen se refiere con este nombre a una carta del 19 de noviembre de 1800 en la que Schelling parece esbozar brevemente un proyecto más amplio y ambicioso que el del Sistema. Este artículo resulta relevante porque es posible establecer, como se concluirá más adelante, un paralelismo entre las tres connotaciones del término "idealismo" en el Systemskizze y sus acepciones en el Sistema. La autora diferencia entre tres sentidos diferentes de "idealismo" que se encuentran en el Systemskizze: a) el primer sentido hace referencia tanto al sistema de Schelling como al de Fichte, es decir, aúna ambos sistemas bajo un proyecto común; ${ }^{21}$ b) el segundo uso del término se emplea para denominar el proyecto sistemático específico de Schelling compuesto de dos partes, una física y una ética, que deberían consumarse en una tercera parte del sistema, es decir, el ideal-realismo o el arte; ${ }^{22}$ c) el último sentido de la palabra es el más específico, designa la parte del sistema que se distingue de la filosofía de la naturaleza y que Schelling llama "ética", "filosofía práctica" y "filosofía trascendental". ${ }^{23}$

La autora reconoce así tres connotaciones diferentes en la carta que podrían biyectarse con las acepciones del Sistema. Un pasaje como este "Si reflexiono sobre las dos a la vez 
[lo subjetivo y lo objetivo], entonces me surge un tercero a partir de ambos, lo que se puede llamar ideal-realismo o lo que hasta ahora hemos designado con el nombre de idealismo trascendental" es congruente con la connotación (b) (nuestro sentido amplio). La sentencia "Aquí no se ha de exponer todo el sistema de la filosofía, sino sólo una de sus ciencias fundamentales" resulta análogo a la connotación (c) de Sandkaulen (nuestro sentido estrecho). Finalmente, la acepción (a) de Sandkaulen (que no tiene correlato en nuestra distinción) podría ser incluída también dentro del llamado sentido amplio (piensese, por ejemplo, en el siguiente pasaje que podría hacer referencia a cierto proyecto común con Fichte: "ampliar el idealismo trascendental a lo que debe ser en realidad, o sea, un sistema de todo el saber"). Este paralelismo permite reflejar el Systemskizze en el Sistema del idealismo trascendental para precisar las diferentes acepciones del término "idealismo" en el Sistema y demostrar que nuestra distinción es convincente.

Hasta aquí nos hemos ocupado de contemplar el problema desde una perspectiva preeminentemente terminológica. Desde este punto de vista parece innegable que Schelling no logra delimitar claramente el alcance de sus expresiones. Distinguimos entre dos sentidos predominantes: el sentido amplio se explica como "ideal-realismo". El segundo sentido fue caracterizado como estrecho. Según éste, el "idealismo trascendental" es una parte de un sistema más amplio y se encuentra en las antípodas de la filosofía de la naturaleza. Si bien no se concluyó qué sentido utiliza Schelling en el título de su obra, la lectura de Fichte en su carta parece sugerir que el Sistema del idealismo trascendental hace referencia al sentido estrecho de la expresión. En efecto, Schelling informa al lector que en la obra no se ha de exponer todo el sistema de la filosofía, sino sólo una de sus ciencias fundamentales. Si esto es así, resulta pertinente preguntar cómo se articula esta ciencia del espíritu con la filosofía de la naturaleza. Es decir, ¿cómo pueden convivir el idealismo trascendental en un sentido estrecho y la filosofía de la naturaleza sin perder su autonomía?

\section{El idealismo trascendental y su articulación dentro del "sistema de todo el saber".}

21 A continuación se intentará mostrar cómo en las reformulaciones posteriores al Sistema Schelling sigue utilizando el concepto de "idealismo trascendental" en dos sentidos diferentes. En segundo lugar, se argumentará que a fin de articular los dos diferentes “idealismos" (la filosofía trascendental y el ideal-realismo), Schelling debe relativizar el papel de la filosofía trascendental. En efecto, para poder convivir, el idealismo en sentido estrecho debe perder protagonismo frente al idealismo en sentido amplio.

Una prueba de que la exposición del concepto de idealismo dentro del Sistema resultó insuficiente hasta para el mismo Schelling lo demuestran las aclaraciones que el autor se ve forzado a hacer en los textos posteriores al Sistema. Aquí nos referiremos concretamente a dos casos: el texto Sobre el verdadero concepto de la filosofía de la naturaleza y Presentación de mi sistema de la filosofía, ambos pertenecientes a 1801.

La preocupación de Schelling por aclarar las confusiones puede examinarse en el texto Sobre el verdadero concepto de la filosofía de la naturaleza (Über den wahren Begriff der Naturphilosophie, de ahora en adelante Sobre el verdadero concepto) de 1801. Allí escribe Schelling con respecto al "idealismo": "Lo que deseo ante todo es que ese término 
adquiera mayor precisión de la que ha tenido hasta ahora". ${ }^{24}$ Si bien allí Schelling se concentra en aclarar los malentendidos que produjo su filosofía de la naturaleza a ojos de Eschenmayer, se pone de manifiesto que el punto de fricción es concretamente la articulación entre idealismo trascendental y filosofía de la naturaleza.

En primer lugar, Schelling se va a preguntar si existe otra manera de filosofar que no sea idealista. ${ }^{25}$ La filosofía sería per se idealismo porque siempre parte del filosofar subjetivo. Esto parecería indicar cierta prioridad por parte del idealismo sobre la filosofía natural. Cuando el sujeto filosofante indaga en la construcción de la filosofía (sea de la naturaleza, sea del espíritu) debe elevarse por medio de la libertad y de la abstracción por sobre el terreno de la individualidad. En un primer sentido, pues, toda filosofía es idealista porque parte de un yo filosofante.

Aunque toda filosofía sea idealista en el filosofar, es decir, en su actividad reflexiva como partiendo de un yo finito que se eleva sobre sus propias condiciones, la filosofía en sí, como sistema del saber, no puede ser idealista en ese sentido. Si lo fuera, no sería científico, porque no habría una salida verdadera del yo de sí mismo, y por lo tanto no habría escisión entre el sujeto y el objeto (lo cual es precisamente lo que Schelling reprocha a la Doctrina de la ciencia). ${ }^{26}$

Frente a "varios filósofos" que presumen entender qué es el idealismo, ${ }^{27}$ Schelling se ve obligado a clarificar su idea: el idealismo no piensa lo real y lo ideal como dos cosas absolutamente separadas. El fundamento del idealismo es la identidad absoluta. La filosofía de la naturaleza parte de lo puramente objetivo, la filosofía trascendental de lo puramente subjetivo. Ambas son idealistas, sin embargo, porque sus respectivos principios son la identidad pura contemplada desde dos perspectivas diferentes. De lo que se sigue, que Schelling parece mantener simultáneamente el sentido amplio y el sentido estrecho de idealismo.

Este idealismo en sentido amplio parece ser el llamado "sistema de la identidad" que Schelling anuncia en el texto aparecido en la Zeitschrift für spekulative Physik de 1801: Presentación de mi sistema de la filosofía (Darstellung meines Systems der Philosophie, en adelante, Presentación). La filosofía, dice allí Schelling, es una, pero ha sido expuesta por él desde dos aspectos diferentes, como filosofía de la naturaleza y la filosofía trascendental. Allí, confiesa, que se ve obligado a presentar ahora "el sistema mismo" antes de lo que él hubiera querido. ${ }^{28}$ No se trata de un cambio de sistema, se empecina en argüir el filósofo, ni de una nueva perspectiva, sino más bien de una perspectiva ya presupuesta en las presentaciones anteriores pero no tematizada. En consonancia con lo ya expuesto Schelling escribe:

"Yo he representado aquello que llamé filosofía de la naturaleza y filosofía trascendental siempre como dos polos contrapuestos del filosofar; con la actual presentación me encuentro en el punto de indiferencia [Indifferenzpunkt] [...].". ${ }^{29}$

En la carta del 24 de mayo de 1801, posterior a la publicación de la Presentación, Schelling reitera un uso amplio del vocablo. Allí dice que el idealismo es el "sol verdadero que abarca, comprende y penetra todo". ${ }^{30}$ ¿Qué es este sol? Aquello que unifica, es decir, la identidad absoluta.

El esquema de lo que tiene pensado Schelling parece estar constituido de tres ámbitos: una filosofía de la naturaleza, una filosofía trascendental y una filosofía de la identidad. A pesar de todo, esta articulación no explica el rol del idealismo dentro de la concepción Schellinguiana. 
30 El idealismo trascendental en sentido estrecho sigue estando opuesto a la filosofía de la naturaleza y eso implica un problema fundamental. Cuando Fichte escribe "[e]n lo que se refiere a la oposición que hace usted [Schelling] entre la Filosofía Trascendental y la Filosofía de la Naturaleza aún no estoy de acuerdo con usted", se señala un problema más profundo que un mero desacuerdo formal. ¿Cuál es la potencial contrariedad que remarca Fichte? Si la filosofía trascendental y la filosofía de la naturaleza son dos ciencias opuestas y autónomas, se pone en peligro la independencia del yo. Dicho de otro modo, hay dos formas de pensar la relación entre filosofía trascendental y filosofía de la naturaleza: o bien las dos se encuentran absolutamente balanceadas en cuanto a su función dentro del sistema; o bien una de las dos tiene una prioridad sobre la otra. La paradoja es que el mismo hecho de que sean autónomas supone que el yo absoluto no puede ser absoluto en un sentido irrestricto.

31 La forma en la que Schelling resuelve esta dificultad no es del todo satisfactoria. Schelling no puede admitir la dependencia del yo (el punto de partida de la filosofía trascendental) frente a la naturaleza, pues eso mermaría su libertad. Sin embargo, Schelling tampoco parece predispuesto a aceptar una libertad absolutamente incondicionada en el Yo. Eso reduciría a la naturaleza a un mero producto secundario. El intento de Schelling para articular el ideal-realismo con el idealismo trascendental consiste en mostrar que el fundamento del idealismo trascendental se encuentra en la identidad absoluta misma. El problema es que para hacer eso, debe privar al Yo de las cualidades que lo caracterizaban: la conciencia y la libertad.

Un primer paso en dirección a lo que podría llamarse relativización del idealismo es su crítica a quienes ven en su filosofía trascendental, un idealismo "ingenuo". Esto es, idealismo no significa pensar al yo empírico e individual como productor de la naturaleza. Esta crítica es, sin duda, ingenua y parece ser trivial. Sin embargo, no es cierto que Schelling la ignore. Por el contrario, ofrece varios argumentos para neutralizarla. Estos razonamientos sugieren, por otra parte, cierto primer debilitamiento de la figura del Yo dentro de su idealismo.

En la denominada "filosofía teórica" del Sistema, Schelling alega que "es bastante manifiesto que realmente todas las fuerzas del universo se retrotraen a fuerzas representativas [...]". ${ }^{31}$ Una frase como ésta es la que permitió ver en el idealismo schellinguiano un correlato directo del idealismo "ingenuo". Si el universo entero surge del autolimitarse y producir del yo, podría ser que el universo no sea sino una “invención” del yo individual y empírico, que existe sólo mientras dura el yo.

Llamamos a esta crítica "ingenua" porque se vale de una comprensión ingenua del idealismo, tal como fue descripta más arriba. Para ésta, todo es representación, todo es un producto del yo. El problema de la interpretación ingenua es que entiende la proposición "toda representación es producida por el yo" como implicando que se trata de un yo individual y empírico. A pesar de ello, es posible preguntarse, si la crítica ingenua tiene su asidero dentro del texto de Schelling.

Por un lado, es claro que en el Sistema Schelling es consciente de esta crítica. ${ }^{32}$ En diferentes pasajes del Sistema, Schelling se refiere a fenómenos como la ceguera, el sueño, ${ }^{33}$ la enfermedad ${ }^{34}$ y la muerte,$^{35}$ es decir, afecciones del yo individual y empírico, y los explica en el marco teórico del idealismo trascendental. Por ejemplo, un crítico ingenuo podría preguntarse "si toda representación es producida por el yo, ¿como es posible que los ciegos no vean? Despues de todo, ellos son los productores de las representaciones". Schelling parece contestar a esta crítica cuando escribe: 
"Si queremos expresarnos de modo trascendente, el ciego de nacimiento, por ejemplo, tiene sin duda una representación de la luz para otro observador aparte de él, ya que para esto sólo se precisa la facultad interna de intuición, pero esta representación no se hace objeto para él [...]." ${ }^{36}$

El yo empírico para Schelling es un órgano de manifestación del Yo absoluto. En el yo empírico es un reflejo del Yo absoluto y de su facultad productiva. Es por ello que el ciego posee una representación de la luz, pero no es objeto para sí mismo. Schelling en ningún momento ignora la crítica del idealismo ingenuo. ${ }^{37}$ Más bien, antes que expulsarla irremediablemente de su planteo, la integra a él, le da un lugar en él.

En Sobre el verdadero concepto, Schelling retoma de forma paródica las críticas “ingenuas" al idealismo. Así satiriza a quienes preguntan: "Aquí hay una árbol que alguien ha plantado hace cincuenta años para la posteridad; ¿cómo es posible que sea yo el que lo esté haciendo aparecer precisamente ahora, tal como es, por medio de la intuición productiva?". ${ }^{38}$

Estas "preguntas embarazosas" (verfänglichen Fragen), por más que Schelling prefiera ignorarlas, aluden, no obstante, a un problema complejo, a saber, ¿cómo es posible una construcción idealista de la naturaleza? En rigor, la pregunta "ingenua" sobre el árbol no es sino una simplificación de esta cuestión: ¿cómo es posible que el Yo como inteligencia produzca los objetos naturales?

Está claro, que el Yo productor de la naturaleza no puede ser el yo empírico. Esto se demuestra por la crítica schellinguiana al yo individual del idealismo ingenuo. Por otro lado, tampoco puede tratarse del Yo fichteano. Fichte, para Schelling, no hace más que permanecer en un Yo subjetivo, incapaz de salir de sí mismo.

Esta idea puede observarse, por ejemplo, en su carta dirigida a Fichte del 19 de noviembre de 1800:

“[...] yo también, como usted, pongo ambas actividades en uno y el mismo Yo, aquí no se encuentra el motivo [de mi oposición entre filosofía trascendental y filosofía de la naturaleza]. El motivo de la misma consiste en que justamente ese Yo como un Yo ideal-real puramente objetivo, y por eso, al mismo tiempo productivo, precisamente en su acción de producir no es otra cosa que Naturaleza, de la cual el Yo de la intuición intelectual o de la autoconsciencia es únicamente la potencia superior". ${ }^{39}$

41 Aquí aparece el Yo pensado como puramente objetivo e inconsciente. Pero resulta lícito preguntar, ¿qué es un yo sin conciencia? ¿En qué se diferencia este yo inconsciente de la naturaleza? El Yo ideal-real puramente objetivo "no es otra cosa que Naturaleza", escribe Schelling. La productividad parece ser posible no en tanto yo consciente, sino en tanto yo inconsciente. La cosa no se añade a la conciencia, no es "algo encontrado" (Gefundenes), la cosa es producida por la conciencia, pero por la conciencia que es naturaleza, es decir, la conciencia-inconsciente. En pocas palabras, la naturaleza es producida por algo análogo a sí misma. ${ }^{40}$ Esta idea sugiere cierta "prioridad" de la filosofía de la naturaleza, la ciencia que parte de lo inconsciente, sobre la filosofía trascendental.

42 Para resolver la pregunta de cómo es posible la producción de la naturaleza a partir del Yo, Schelling tiene que transformar a este Yo en un Yo-inconsciente. Es por eso que la crítica al idealismo ingenuo parece ser un primer paso en la dirección de debilitar al yo. Este debilitamiento del Yo, parece venir de la mano de cierta prioridad de la filosofía de la naturaleza. En Sobre el verdadero concepto el autor sentencia que existe "un idealismo 
de la naturaleza y un idealismo del Yo. El primero es para mí el originario y el segundo el derivado". ${ }^{41} \mathrm{Y}$ un poco más adelante escribe:

"La ventaja de la filosofía de la naturaleza con respecto al idealismo es que ésta demuestra de modo puramente teórico sus proposiciones pudiendo prescindir de todo tipo de exigencias especiales o prácticas, mientras que el idealismo no puede, motivo por el que no tiene ninguna realidad teórica pura, tal como he señalado en el prólogo al Sistema del idealismo." ${ }_{42}$

Y el pasaje al que se refiere Schelling del Sistema señala precisamente que:

“[...] el idealismo tampoco tiene un fundamento puramente teórico y, por tanto, si sólo se admite la evidencia teórica, nunca podrá tener la evidencia de la que es capaz la ciencia de la naturaleza, cuyo fundamento, igual que sus pruebas, son total y absolutamente teóricos." ${ }^{43}$

Ciertamente aquí se trata de una prioridad metodológica: La intuición intelectual de la filosofía trascendental no puede ser nunca absolutamente teórica porque el filósofo nunca puede abstraer absolutamente su propio yo del Yo puro. Ambos permanecen, al menos en la práctica, unidos porque no se puede hacer abstracción del que intuye: "no lleva a cabo la abstracción del que intuye, la cual sin embargo es necesaria cuando se quiere instaurar una filosofía puramente objetiva [...]" ${ }^{44}$ Por eso, dice el filósofo, el yo como principio de la parte trascendental debe pensarse como un yo inconsciente, a fin de ser lo más teórico posible. ${ }^{45} \mathrm{El}$ principio de la filosofía de la naturaleza, el puro ser, en cambio, es más abstracto en el sentido de que no está relacionado con el yo filosofante de ningún modo. El idealismo es la ciencia fundamental que busca deducir a partir del sujeto-objeto de la conciencia el sujeto-objeto puro en sí (esto es, la naturaleza). De esta manera Schelling afirma la prioridad (Priorität) de la filosofía de la naturaleza, pues, "es ella la que hace surgir el punto de vista del propio idealismo y de este modo le procura una base segura y puramente teórica". ${ }^{46}$ En ese caso, sin embargo, la ventaja de la filosofía de la naturaleza dejaría de ser metodológica para ser también ontológica: habría una preponderancia de lo inconsciente sobre lo consciente.

En conclusión, si bien Schelling desestima las "preguntas embarazosas" de la crítica al idealismo ingenuo, no puede escapar a dar una respuesta de cómo es posible una construcción de la naturaleza (pregunta, en el fondo, incluida en aquellas otras) a partir del yo. Para ello parece tener que relativizar el papel del idealismo dentro de su sistema. Para articular el idealismo en sentido amplio con el idealismo en sentido estrecho, el autor debe subordinar el segundo al primero. Por otro lado, esto parece modificar la relación entre la filosofía trascendental y la filosofía de la naturaleza. Si bien ambas ciencias son metodológicamente independientes y ambas pueden desenvolverse sin el auxilio de las otras, la filosofía de la naturaleza precede, al menos desde un punto de vista teórico, al idealismo. Para no converger con un mal idealismo ingenuo, se debe apelar a un momento pre-reflexivo del yo que no hace más que reducirlo a un momento de la naturaleza en tanto natura naturans (el momento más alto, si se quiere). Y si bien ambas filosofías son "idealistas" en un sentido amplio porque ambas reposan sobre la identidad absoluta, la filosofía trascendental es "idealista" en un sentido propio porque lleva a cabo su deducción a partir del puro sujeto-objeto de la conciencia, el Yo. El idealismo se posiciona así como contrapartida de la filosofía de la naturaleza, como un compañero necesario y al mismo tiempo dependiente de esta última ciencia. 


\section{Conclusión}

Frente a las preguntas que nos formulamos podemos concluir algunas proposiciones. Primero: el concepto de "idealismo trascendental" no encuentra una exposición unívoca en el Sistema. Se distinguió entre un sentido estrecho y un sentido amplio. Prueba de esta polisemia no es sólo la bibliografía actual (como el citado artículo de Sandkaulen) sino también el intercambio epistolar de Schelling con Fichte y los posteriores intentos del autor del Sistema por aclarar el término (en Sobre el verdadero concepto y Presentación).

Segundo: entre los dos sentidos de "idealismo trascendental" se engendra una tensión. Filosofía trascendental y filosofía de la naturaleza se articulan como dos ciencias opuestas en el marco del sistema de todo el saber, llamado por Schelling "idealrealismo", "sistema de la identidad", etc. El fundamento de la consciencia y de la naturaleza reposa en la identidad absoluta. Esto relativiza el poder explicativo de la filosofía trascendental. En efecto, ésta parece no ser capaz de explicar el surgimiento de la naturaleza sino es apelando a una instancia que Schelling llama "Yo ideal-real puramente objetivo". Este Yo, sin embargo, al ser un principio inconsciente, se muestra más cercano al concepto de naturaleza que al concepto de un yo ligado a la libertad absoluta.

Schelling efectivamente parece relativiza la posición del idealismo frente a la filosofía de la naturaleza. No sólo relativiza el papel del idealismo (que ya no es el único protagonista dentro del proyecto schellinguiano), sino que también deja al descubierto las falencias de todo idealismo que no asuma seriamente el problema de la ontología (es decir, de la naturaleza).

El Sistema escenifica así una tensión inmanente al concepto mismo de "idealismo". "Idealismo trascendental", hace referencia a un idealismo productivo no ingenuo. Se trata de un idealismo absoluto pero sólo en la medida en que lo absoluto fagocita el idealismo, en la medida en que las fuerzas productivas inconscientes obran sobre la consciencia a la vez que la generan. Al criticar el idealismo subjetivo de Fichte, Schelling parece optar por una salida ontológica de la pregunta del Sistema. ¿Cómo es posible una construcción idealista de la naturaleza? La respuesta de Schelling parece ser: es posible en la medida en que el idealismo y la naturaleza comparten una estructura productiva. La identidad absoluta es la savia que permite a una y a otra obrar productivamente. Esta identidad absoluta no es realista ni idealista, sino idealrealista y real-idealista. De cualquier manera, siempre es lícito volver a preguntar, ¿no termina necesariamente uno de los polos por fagocitar al otro?

Por estos motivos, es pertinente colegir que la dificultad de hermanar el término "idealismo" a un sólo sentido no es accidental. Por un lado, da cuenta de una confusión inmanente al mismo "proyecto idealista" de Schelling y Fichte. Por otro lado, señala la tensión interna de Schelling y coloca al Sistema en un punto de transición hacia la llamada filosofía de la identidad. Finalmente, subraya el dinamismo del concepto de "idealismo" que se revela como una idea lo suficientemente vasta como asimilar diferentes significados. 


\section{Bibliografía}

FICHTE, J.G. \& SCHELLING F.W.J., (1856) Fichtes und Schellings philosophischer Briefwechsel. Stuttgart/Ausburg: Cotta. [=FSpB]

FICHTE, J.G., (1984) Introducción a la Teoría de la Ciencia. Madrid: Sarpe. [=IntroDC] HÜHN, L., (2005) "Die Verabschiedung des subjektivitätstheoretischen Paradigmas", FICHTE-STUDIEN. GRUNDLEGUNG UND KRITIK. DER BRIEFWECHSEL ZWISCHEN SCHELLING UND FICHTE 1794 - 1802, 93-111.

KISSER, T., (2011) "Unbestimmtheit und Unbedingtheit. Einige Anmerkungen zu Fichtes Kritik an Schelling um 1800 und der Entwicklung des Schellingschen Denkens", en: Danz, C. \& Stolzenberg, J. (eds.) System und Systemkrititik um 1800. Hamburg: Meiner, 193-209.

KISSER, T., (2013) "Schellings Spinozismus", SCHELLING STUDIEN 1, 157-186. REINHOLD, K.L., (1801) Beyträge zur leichtern Übersicht des Zustandes der Philosophie beym Anfange des 19. Jahrhunderts. Bd. 2. Hamburg: Perthes.

SANDKAULEN, B., (2005) "Was heißt Idealismus?", FICHTE-STUDIEN. GRUNDLEGUNG UND KRITIK. DER BRIEFWECHSEL ZWISCHEN SCHELLING UND FICHTE 1794 - 1802, 57-70.

SCHELLING, F.W.J., Las citas a la obra de Schelling se refieren a la edición de 1856-1861: F.W.J. Schelling: Sämmtliche Werke. Stuttgart Cotta. [=SSW]

----, (1988) Sistema del idealismo trascendental. Traducción Jacinto Rivera de Rosales y Virginia López Domínguez. Barcelona: Anthropos.

----, (1993) Cartas sobre dogmatismo y criticismo. Traducción Virginia Careaga. Madrid: Tecnos.

----, (1996) Escritos sobre filosofía de la naturaleza. Traducción Arturo Leyte. Madrid: Alianza.

SCHWENZFEUER, S., (2012) Natur und Subjekt. Die Grundlegung der schellingschen Naturphilosophie. Freiburg/München: Karl Alber.

SERRANO MARÍN, V., (2008) Absoluto y conciencia. Madrid: Plaza y Valdes. STOLZENBERG, J., (2011) "Der Streit ums Absolute", en: Danz, C. \& Stolzenberg, J. (eds.) System und Systemkrititik um 1800. Hamburg: Meiner, 181-192.

\section{NOTAS}

1. Las citas a la obra de Schelling se refieren a la edición de 1856-1861: F.W.J. Schelling: Sämmtliche Werke. Stuttgart Cotta [=SSW]. Además de las abreviaturas convencionales utilizaremos FSpB para referirnos a la correspondencia editada por Cotta Fichtes und Schelling philosophischer Briefwechsel (1856) y SAA para referir a la F. W. J. Schelling, Historisch-kritische Ausgabe (Akademie Ausgabe) editada por H. Baumgartner entre otros. Al menos que se aclare lo contrario, las traducciones son mías.

2. Pienso aquí principalmente en los trabajos de Sandkaulen (2005), Hühn (2005), Stolzenberg (2011), Kisser $(2011 ; 2013)$ y Schwenzfeuer (2012). Esta tensión emerge siempre de la mano del distanciamiento entre Fichte y Schelling. El motivo no es 
accidental: como señala Sandkaulen, la oposición entre filosofía trascendental y filosofía de la naturaleza es el "nervio central" de la ruptura entre Fichte y Schelling (Sandkaulen 2005: 59). Hühn explica el enfrentamiento con vistas a una "despedida del paradigma subjetivo-teórico" (Hühn 2005). En los textos más recientes las insuficiencias del planteo de Schelling se explicitan. Stolzenberg afirma que "el programa de Schelling de fundamentar la filosofía trascendental y la filosofía de la naturaleza como dos discursos inversos y al mismo tiempo complementarios no es sostenible" (Stolzenberg 2011: 184). Kisser se refiere a la "aporía" que implica pensar la unidad de la actividad inconsciente y consciente (Kisser 2011: 203) y en su texto sobre el espinozismo de Schelling vuelve a señalar la "paradoja" entre la libertad del sujeto y la necesidad del objeto (Kisser 2013: 172). Si bien Schelling ofrece una solución por medio del arte, ésta no deja de ser débil. En efecto, resulta pertinente preguntar, ¿es lícito que la filosofía delegue la consumación del sistema al arte? (Kisser 2013: 174). Por su parte, Schwenzfeuer declara que la superación de la filosofía trascendental por parte de la filosofía de la identidad se debe más a una necesidad interna del idealismo que a la crítica aludida por Fichte acerca de la imposibilidad de dos ciencias fundamentales (Schwenzfeuer 2012: 151). Sea como fuere, quedan al descubierto no sólo las tensiones del Sistema sino incluso sus deficiencias.

3. SSW I/1, 330; trad. Schelling 1993: 86.

4. "Cada uno [de estos dos sistemas] niega todo al opuesto, y no tienen absolutamente ningún punto en común, desde el cual pudieran entenderse el uno al otro y ponerse de acuerdo" (EE, GA I/4, 191; IntroDC, 40). Sobre este disentimiento ver también la carta de Fichte a Schelling del 31 de mayo de 1801 (FSpB, 81).

5. Cfr. SSW III/1, 427; trad. Schelling 1988: 231.

6. Cfr. SSW III/1, 343; trad. Schelling 1988: 153.

7. Cfr. SSW, III/1, 356; trad. Schelling 1988: 164.

8. Cfr. EE, GA I/4, 195; IntroDC, 46.

9. SSW, III/1, 256; trad. Schelling 1988: 165.

10. SSW III/1, 386; trad. Schelling 1988: 194.

11. SSW III/1, 330; trad. Schelling 1988: 138.

12. SSW III/1, 342-343; trad. Schelling 1988: 152.

13. SSW III/1, 342-343; trad. Schellin, 1988: 152.

14. FSpB, 54.

15. Serrano Marin 2008: 229

16. Reinhold 1801: 68.

17. Ver su recensión del Primer esbozo de un sistema de la filosofía de la naturaleza aparecida en el Erlanger Literatur-Zeitung en abril de 1801. Citado en SAA III 2(2), 706-707.

18. FSpB, 58.

19. Sin confundirla, claro, con el ideal-realismo concreto y objetivo del arte (que se encuentra al final del Sistema pero que concretamente no forma parte de él). (FSpB, 60) 20. Cfr. Sandkaulen 2005: 61.

21. Piénsese en el siguiente pasaje del Systemskizze: "Lo que me resulta muy importante [escribe Schelling a Fichte], antes de coincidir en hacer algo juntos, es nuestra conformidad sobre algunos puntos que Ud., en parte, menciona en su carta y que son de suma importancia para el Idealismo, tal como yo lo entiendo y siempre lo entendí". $(\mathrm{FSpB}, 57)$ 
22. Compárese con el siguiente fragmento: “el idealismo transcendental vale solamente para quien se haya propuesto originalmente partir del saber en su potencia máxima, en tanto que es un saber teorético y práctico al mismo tiempo". (FSpB, 62)

23. Cfr. Sandkaulen 2005: 61.

24. SSW IV/1, 84; trad. Schelling 1996: 260.

25. Cfr. SSW IV/1, 84; trad. Schelling 1996: 260.

26. Cfr. SSW IV/1, 85-86; trad. Schelling 1996: 261.

27. Cfr. SSW IV/1, 87; trad. Schelling 1996: 262.

28. Cfr. SSW IV/1, 107. Ver también, Sobre el verdadero concepto: SSW IV/1, 89; trad. Schelling 1996: 264.

29. SSW IV/1, 108.

30. FSpB, 77.

31. SSW III/1, 487; trad. Schelling 1988: 287.

32. "Una objeción muy frecuente contra el idealismo es que las representaciones de las cosas externas nos llegan de forma completamente involuntaria." (SSW III/1, 485; trad. Schelling 1988: 286)

33. "En el sueño, por ejemplo, se suprime el producir originario, es la reflexión libre la que se interrumpe junto con la conciencia de la individualidad." (SSW III/1, 506; trad. Schelling 1988: 306)

34. “[...] [A]penas un individuo deja de ser un perfecto reflejo de nuestro universo, deja también de servir como órgano de autointuición, es decir, está enfermo." (SSW III/1, 498; trad. Schelling 1988: 298)

35. "Yo, en cuanto soy este individuo determinado, no era en absoluto antes de intuirme como tal, ni seré el mismo tan pronto como esta intuición cese." (SSW III/1, 498; trad. Schelling 1988: 299)

36. SSW III/1, 497; trad. Schelling 1988: 297.

37. Veáse también el siguiente pasaje: “Una cuestión que el idealista no puede evitar es la de cómo llega a admitir un pasado o qué se lo garantiza. Lo presente se lo explica cada uno a partir de su producir; pero ¿cómo llega a admitir que algo era antes de que él lo produjera?" (SSW III/1, 498; trad. Schelling 1988: 287)

38. SSW IV/1, 83; trad. Schelling 1996: 259.

39. FSpB, 58.

40. En la carta del 31 de mayo de 1801 Fichte insiste en que "la deducción de una naturaleza a partir de la inteligencia y, otra vez, de la inteligencia a partir de la inteligencia" implica un círculo lógico demasiado obvio como para que Schelling no lo repare (FSpB, 82). Schwenzfeuer remarca una pertinente distinción: en rigor, es incorrecto afirmar que nos encontramos frente a un círculo lógico. La naturaleza que surge del yo en el marco de la filosofía trascendental no es la misma que aquella que constituye la pre-historia inconsciente del yo. La primera es natura naturata, es producto de la facultad productiva del yo como principio de la filosofía trascendental. La natura naturans de la que depende ontológicamente ese yo, por su parte, no puede ser deducida de una instancia superior (Cfr. Schwenzfeuer 2012: 154). Esta distinción es correcta pero en última instancia no salda las insuficiencias del idealismo. Sea que se lo subsuma a una natura naturata (dogmatismo ingenuo o materialismo) o a una natura naturans (filosofía de la naturaleza, ¿filosofía de la identidad?), su destino es el mismo.

41. SSW IV, 84; trad. Schelling 1996: 260.

42. SSW IV, 91; trad. Schelling, 1996: 266.

43. SSW III/1, 332; trad. Schelling 1988: 140. 
44. SSW IV, 90; trad. Schelling 1996: 265.

45. Cfr. SSW IV, 88.

46. SSW IV/1, 91; trad. Schelling 1996: 267.

\section{RESÚMENES}

In this paper I will investigate the concept of "transcendental idealism" in the System of Transcendental Idealism (1800). My aim is to outline the difficulties surrounding the concept. This paper argues that there is a marked tension between the different senses of the expression "transcendental idealism" and that in order to solve this tension Schelling tends to relativize the role of idealism in his system. In furtherance of this, I read this work of Schelling in the context of other texts, in particular, On the True Concept of the Philosophy of Nature and the Presentation of My System of Philosophy, both of 1801 .

\section{ÍNDICE}

Keywords: Fichte, philosophy of nature

\section{AUTOR}

\section{FERNANDO WIRTZ}

Universidad de Buenos Aires/Eberhard Karls Universität Tübingen 